\title{
Stream segregation of narrow-band noise bursts
}

\author{
ALBERT S. BREGMAN, PIERRE A. AHAD, and CHRISTINA VAN LOON \\ McGill University, Montreal, Quebec, Canada
}

\begin{abstract}
Normally hearing adults heard rapid alternations of a pair of band-limited noise bursts that had flat spectra (in terms of equal-loudness weighting of components) and sharp band edges. The bursts differed in center frequency (CF), but were matched on overall intensity, on bandwidth (BW) on a logfrequency scale, and (roughly) on pitch strength. Listeners judged the ease with which the sequence could be held together perceptually in a single auditory stream (vs. forming separate high and low streams). Involuntary segregation was examined as a function of the following measures of frequency separation of the alternating noise bands: (1) the closest band edges, (2) the most remote band edges, (3) the CFs of the bands on a logarithmic scale, and (4) the BWs. Segregation was best predicted from the separation of the two CFs on a log-frequency scale (very strong effect). Increasing the BWs of the two alternating bursts (the same size, in log frequency, for both bands) also led to greater segregation (very weak effect).
\end{abstract}

Periodic sounds (tones) represent only a fraction of the sounds that populate our everyday lives. However, most of the research on the perceptual organization of sounds has used tones as stimuli (Bregman, 1990, chap. 2). There are only two studies, to our knowledge, that have addressed the perceptual grouping of noises. The stream segregation of alternating narrow-band noises of higher and lower center frequencies (CFs) was studied by Dannenbring and Bregman (1976) in an experiment that was primarily about the subjective overlap of segregated streams. Their sharply peaked noise bands, created by the filtering of white noise, had two different CF separations, 3.2 and 19.0 semitones. Ratings of stream segregation were higher in sequences that had the 19-semitone separation. Bregman, Colantonio, and Ahad (1999) studied the segregation of band-limited noise bursts of high and low CFs as part of an experiment whose purpose was to demonstrate that several variables (bandwidth [BW], rate of onsets, and separation in CF of the high- and low-pitched noises) would have similar effects on stream segregation and on the continuity illusion ("apparent continuity"). They used noise bands whose spectra were flat between the band edges. Among their other findings, they found that BW had a significant effect on the segregation of narrow-band noises. The present experiment followed up on this experiment, studying the stream segregation of the same types of noise bursts, but controlling their properties more precisely.

Some terminology can be introduced with the help of Figure 1. Two bands of narrow-band noise (NBN) are

This research was carried out with support for salaries from Grant R01 MH52254-03 from the NIMH to A.S.B. Equipment and technical support came from a grant to A.S.B. from the Natural Sciences and Engineering Research Council of Canada. Correspondence should be addressed to A. S. Bregman, Department of Psychology, McGill University, 1205 Docteur Penfield Ave., Montreal, PQ, Canada H3A 1B1 (e-mail: bregman@hebb.psych.mcgill.ca). shown. The one that is higher in frequency is referred to as $\mathrm{H}$, and the lower as $\mathrm{L}$. The top edge of $\mathrm{H}$ and the bottom edge of $\mathrm{L}$ can be called the "outer band edges" of the NBN pair. They represent the two most separated frequency components of $\mathrm{H}$ and L. Similarly, the bottom edge of $\mathrm{H}$ and the top edge of $\mathrm{L}$ represent the two "inner band edges" of the pair, the closest frequencies of the two bands. The CF of each band of noise (on a log-frequency scale) and the BW is also shown, bandwidth being defined here as the difference in frequency between the upper and lower band edges in semitones (a log-frequency scale).

Any such flat-spectrum NBNs, in which all frequencies have equal intensities when plotted on a linear scale of frequency, can be described by a pair of parameters. One possible pair consists of the CF and BW. An alternative description is given by a different parameter pairupper band edge and lower band edge. Although the two descriptions are fully equivalent, they emphasize different properties of the band. The first treats the noise band as a block whose overall frequency can be represented by its $\mathrm{CF}$, whereas the second focuses on the frequencies at the edges.

The experiment by Bregman et al. (1999) showed that the segregation of a sequence into separate $\mathrm{H}$ and $\mathrm{L}$ streams was increased by the difference between the CFs of the two bands of noise. In addition, they found that $\mathrm{H}$ and $\mathrm{L}$ bursts having greater BWs were segregated more than those with smaller BWs. This could not have resulted from the way in which BW affected the separation of the CFs themselves, since the variation of the latter was manipulated orthogonally with BW, but BW did affect the separation of the inner and outer band edges of the $\mathrm{H}$ and $\mathrm{L}$ bands. It is possible that it was the position of these edges rather than the BW per se that affected segregation.

On the other hand, the effects of BW observed by Bregman et al. (1999) may have resulted from a decision they made about how to equate the BWs of different CFs. In 


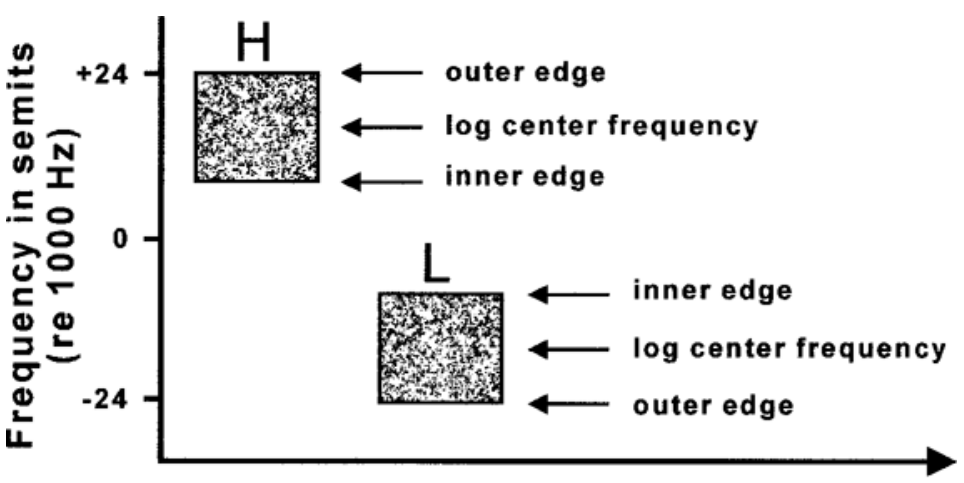

\section{Time}

Figure 1. Outer band edges, inner band edges, and log center frequency for a pair of bursts of narrow-band noise. A higher one $(H)$ and a lower one $(L)$ were rapidly alternated to induce auditory stream segregation. The ordinate represents semitones above $(+)$ and below $(-) 1000 \mathrm{~Hz}$.

pretesting, they had noticed that if noise bands of different CFs had equal BWs, the higher one exhibited greater "pitch strength," sounding more pitchlike and less noisy. They further observed that if the BWs were adjusted according to the size of the critical band at the CF of the band (i.e., keeping constant the ratio of BW to critical band), the $\mathrm{H}$ and $\mathrm{L}$ bands seemed to have approximately equal pitch strengths. Because they believed that differences in pitch strength between $\mathrm{H}$ and $\mathrm{L}$ bands might affect streaming, they equated the $\mathrm{H}$ and $\mathrm{L}$ bands on pitch strength rather than BW per se. Thus the actual bandwidths varied as a function of CF. An unavoidable consequence of this was that higher bands, having greater BWs, were actually more intense and sounded louder than lower bands. Because of other constraints in that experiment (the need to use the same signals to study stream segregation and apparent continuity), the $\mathrm{H}$ and $\mathrm{L}$ bands could not be equalized for intensity. It has been reported by Van Noorden (1975) that intensity differences between alternating tones promoted their segregation into separate streams. If this result can be extrapolated to the case of NBNs, some of the effects of CF separation found by Bregman et al. might be explained by intensity differences. Furthermore, it is possible that the overall BW effects (greater segregation when the alternating $\mathrm{H}$ and $\mathrm{L}$, as a pair, showed greater BW) was an indirect product of the correlation between changes in BW and changes in intensity.

In the present experiment, we tried to keep both the pitch strengths and the loudnesses of the $\mathrm{H}$ and $\mathrm{L}$ bands the same. To deal with pitch strength differences, the BWs of the $\mathrm{H}$ and $\mathrm{L}$ bands were equated in terms of number of semitones. This was done because the semitone is a $\log$-frequency measure-one semitone separation between frequencies is approximately equal to a frequency ratio of 1.06 - and critical bands are also approximately equal in log frequency in a large part of their range. Therefore, if the pitch strength of a noise band that is transposed up and down along a critical-band scale remains fairly constant, it should also remain approximately constant on a log-frequency scale. So if BWs are equal on a semitone scale, the NBNs should be approximately equal in pitch strength. Preliminary listening by the experimenters confirmed that this was the case.

To equate for loudness, first the physical intensities of all NBNs were equated (on RMS amplitude for the entire burst) by digital synthesis. Then, at the time of playback, they were passed through a graphic equalizer to impose a compensation for the effects of frequency on perceived loudness. So while the resulting bands were no longer flat in their physical spectra, they would be flat if plotted on a loudness scale. The present experiment asked how well the following measures predicted stream segregation: (1) separation of CFs, (2) separation of inner band edges, and (3) separation of outer band edges.

\section{METHOD}

\section{Listeners}

The listeners were initially 11 women and 14 men, drawn from a university population. The results from 1 man were discarded due to apparatus problems during testing, leaving 24 listeners. All underwent a short hearing test to ensure that they could hear pure tones in the same frequency range as the NBN stimuli $(250-4000 \mathrm{~Hz})$ at intensities equal to or less than $15 \mathrm{dBA}$. None were rejected by this criterion.

\section{Stimuli}

The NBNs were noise bursts, $65 \mathrm{msec}$ in duration, including 10msec onset and offset ramps that were linear changes in amplitude. The bursts were created by the summation of a large number of closely spaced sinusoidal components in random phases. The frequencies of these components were spaced by $1 \mathrm{~Hz}$ starting from the lower band edge to the upper band edge. For example, a noise band running from 4 semitones above $1000 \mathrm{~Hz}(1260 \mathrm{~Hz})$ to 8 semitones above $1000 \mathrm{~Hz}(1587 \mathrm{~Hz})$ is generated by the addition of 328 frequency components of equal amplitude and random phase. This was exactly the same method as was used by Bregman et al. (1999).

Higher $(\mathrm{H})$ and lower $(\mathrm{L}) \mathrm{NBNs}$ were presented in a cycle and played in a galloping pattern whose structure can be described by 
the letter sequence, HLH-HLH-.... The dash in the letter sequence "HLH-" represents a 100-msec silence (as if an L and the subsequent interburst silence had been omitted). When stream segregation takes place, the listener hears two streams, one formed of the $\mathrm{H}$ bands $(\mathrm{H}-\mathrm{H}-\mathrm{H}-\mathrm{H}-\ldots)$ and the other formed of the $\mathrm{L}$ bands, repeating at half the rate $(\mathrm{L}-\mathrm{L}-\ldots)$.

The 400-msec pattern (HLH-) was repeated 12 times, for a total trial duration of $4.8 \mathrm{sec}$, with a linear fade-in of amplitude over the first three cycles. Each "HLH-" triplet was timed as follows: 65msec $\mathrm{H}$ burst, 35-msec silence, 65-msec L burst, 35-msec silence, 65-msec H burst, 35-msec silence, 100-msec silence. Hence the duration of each "HLH-" triplet was $400 \mathrm{msec}$. The stimulus onset asynchrony (SOA) for successive $\mathrm{H}$ bursts (ignoring the $\mathrm{L}$ bursts) was $200 \mathrm{msec}$, whereas the SOA for the L noises taken alone was $400 \mathrm{msec}$. The high and low NBNs were placed symmetrically around $1000 \mathrm{~Hz}$ on a semitone (log-frequency) scale.

Table 1 shows the descriptions of all the NBNs used in the experiment, expressed in semitones above $1000 \mathrm{~Hz}$. Semitones form a logarithmic scale; one semitone difference between two tones (which corresponds to the difference between the notes $\mathrm{C}$ and $\mathrm{C} \#$ in Western music) corresponds to a ratio of 1.06 between them. Only the values for the $\mathrm{H}$ bands are given, since the $\mathrm{L}$ bands were placed symmetrically below $1000 \mathrm{~Hz}$, on a semitone scale. We used negative numbers to represent values below $1000 \mathrm{~Hz}$. Therefore the displacements of the $\mathrm{L}$ bands from $1000 \mathrm{~Hz}$ are the negative of those shown in the table. The parameters are outer band edge (e.g., the upper edge of the $\mathrm{H}$ band), $\mathrm{CF}$, and inner band edge (e.g., the lower edge of the $\mathrm{H}$ band). For each condition, we also show the results of the experiment - the mean rated segregation on a 1-7 scale, with higher numbers indicating greater segregation. Standard errors are shown in parentheses.

When a negative value is given for the frequency of the inner $\mathrm{H}$ band, this indicates that it was below $1000 \mathrm{~Hz}$, so that the $\mathrm{H}$ band partially crossed the $1000-\mathrm{Hz}$ midpoint. Since the corresponding L band crossed the midpoint in a symmetrical manner, this meant that there was overlap of the frequencies of $\mathrm{H}$ and $\mathrm{L}$ bands. The separations (or overlaps) of the $\mathrm{H}$ and $\mathrm{L}$ bands were twice the values shown in Table 1 and Figure 2, since the deviations of the $\mathrm{H}$ and $\mathrm{L}$ bands from $1000 \mathrm{~Hz}$ must be added together to determine the $\mathrm{H}-\mathrm{L}$ separation. Therefore the actual separations, shown in Figure 2, are twice those shown in Table 1.

Three series of conditions were designed: A, B, and C. Each varied a different description of frequency separation orthogonally with BW. The first part of Table 1 shows the frequency parameters for Series A, in which the outer band edge and $\mathrm{BW}$ were varied orthogonally. Since each value of the outer band edge was held constant while $\mathrm{BW}$ varied, the variation in $\mathrm{BW}$ forced a corresponding variation in the $\mathrm{CF}$ and the inner band edge. BWs were $16,12,8$, and 4 semitones. The outer band edges (distances between extreme edges of the $\mathrm{H}$ and $\mathrm{L}$ bands) were separated by $48,42,36$, and 30 semitones. These values were used in Series A (Figure 2).

The second part of Table 1 shows the frequency parameters for a second series of conditions (Series B). This time the inner band edge and BW were varied orthogonally. In this series, it was the changes in the separation between the outer band edges of $\mathrm{H}$ and $\mathrm{L}$ bands that produced the different BW levels, pulling the CFs along with them. BWs were $16,12,8$, and 4 semitones. Inner band edges were -2 (overlapped by 2), 4, 10, and 16 semitones apart. These values were used in Series B (Figure 2).

The third part of Table 1 shows the frequency parameters for a third series of conditions (Series C). This time, $C F$ and $\mathrm{BW}$ were varied orthogonally, with each CF held constant across the four levels of BW. In this case it was the symmetrical variations in the upper and lower edges that produced the different BW levels. BWs were $16,12,8$, and 4 semitones. CFs were $6,12,18$, and 24 semitones apart. These values were used in Series C (Figure 2).

\section{Procedure}

The task of the listeners was to try to "hold on to the simple melody" (i.e., the galloping triplet pattern). To do this, they had to hear the sequence as integrated. At the end of a trial they rated their difficulty on a 7-point scale, from 1 (very easy) to 7 (very difficult). When listeners are asked to try to hold on to the sequence as a whole, this can be done only when the frequency separations are below Van Noorden's (1975) “temporal coherence boundary.” It is this boundary that is known to be affected by both the frequency separation and the speed of pure tones. We assumed that the same would hold true for NBNs.

In a preliminary training period, stream segregation was explained to the listeners by way of pictures and auditory demonstrations, employing NBNs that unambiguously led to one-stream or two-stream percepts. It was verified that all listeners understood the distinction between segregation and integration. Then they were further trained by being run once through all 48 experimental conditions in a randomized order so they could adjust their use of the rating scale to the range of percepts and become familiar with the procedure. Then, in the experiment proper, listeners completed two blocks of 48 trials. Each block presented all 48 conditions in a random order. There was a new randomization of order for each block and each listener.

\section{Apparatus}

The signals were synthesized with the use of MITSYN 8.1 signalprocessing software (Henke, 1990) at 20,000 samples per second, output via a 16-bit D/A converter, and low-pass filtered through a passive Chebyshev filter having a $3-\mathrm{dB}$ cutoff at $8 \mathrm{kHz}$ and a falloff of approximately $142 \mathrm{~dB} /$ octave. Listeners were tested individually in an audiometric chamber. The sounds were presented binaurally over Sennheiser HD-414 headphones. The preliminary hearing test used a Maico Instruments Model MA 27 Series 101 tone generator on the pulse setting.

\section{Intensity}

The RMS amplitudes of the NBNs in the experiment were equated by the synthesis. The intensity correction was applied to each NBN as a whole, and therefore equally to all its frequency components so that, at this stage, the band remained flat in amplitude. At the time of playback, all intensities were measured using a flatplat coupler between the headphones and an SPL meter. The playback intensity was calibrated by setting to $60 \mathrm{~dB}$ (B weighting) a long version of the noise burst centered at $1000 \mathrm{~Hz}$, with a BW of 16 semitones $(749-1888 \mathrm{~Hz})$. After amplification, the signals passed through an analog graphic equalizer to impose an equal-loudness compensation based on the Fletcher-Munson curve at $60 \mathrm{~dB}$, the level at which the sounds would be presented. (This curve shows the intensity boost for a pure tone at various frequencies required for it to sound as loud as a $60-\mathrm{dB}$ pure tone at $1000 \mathrm{~Hz}$.) Imposing this compensation caused the spectra to no longer be exactly flat in amplitude; however, they were intended to be approximately flat in terms of the perceived loudness of their components.

\section{RESULTS}

Since this was not a fully orthogonal design, it was impossible to analyze all the data at once. Consequently, mean rating data for Series A, B, and C (Table 1 and Figure 2) were analyzed separately as two-way, withinsubjects designs using the multivariate analysis of variance (MANOVA) model.

\section{Series A}

Analysis of the outer band edge by BW (first part of Table 1 and top panel of Figure 2) showed highly signif- 


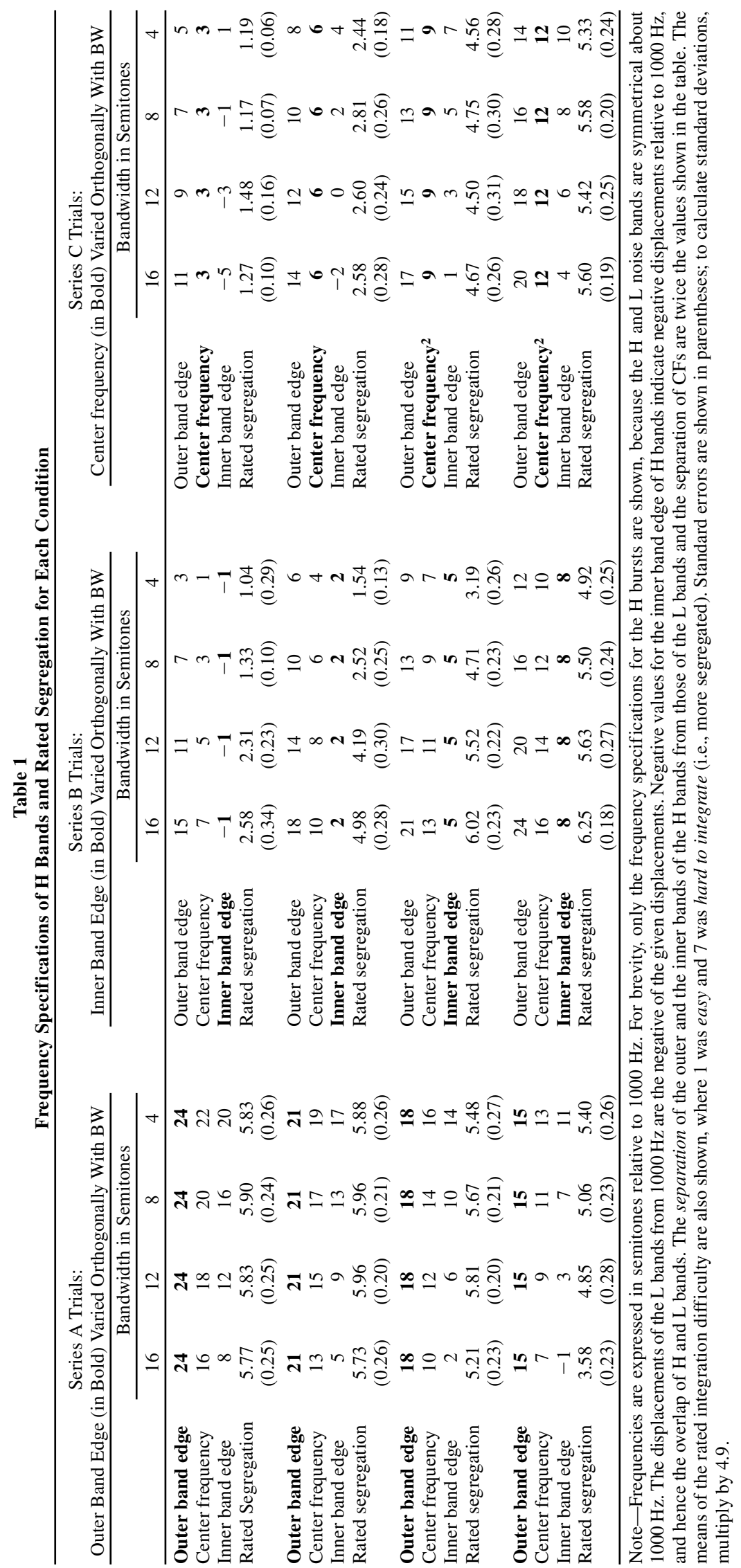



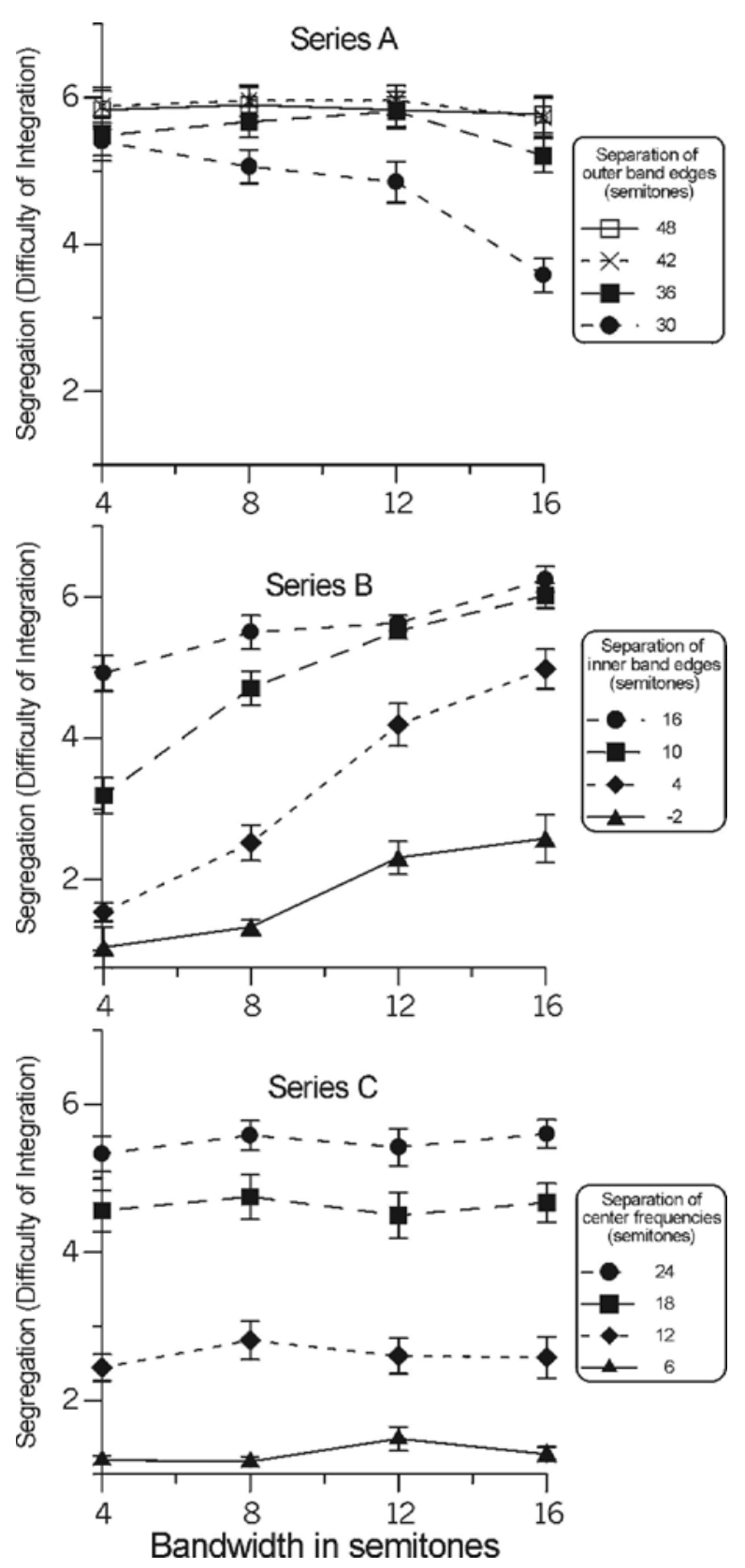

Figure 2. Segregation, as measured by rated difficulty of integration $(1=$ easy, $7=$ hard $)$, is plotted as a function of bandwidth for Series A trials (systematic variation in outer band edge), Series $B$ trials (systematic variation in inner band edge), and Series $C$ trials (systematic variation in center frequency). Each point represents the mean for all listeners for 1 of the 48 experimental conditions. High scores represent compelling segregation. Frequency separations are expressed in terms of the separation in semitones of $\mathrm{H}$ and $L$ bursts. Hence these values are twice those in Table 1, which shows their separation from the spectral midpoint of $1000 \mathrm{~Hz}$.

icant effects of outer band edge $[F(3,69)=33.0, p<$ $.00001]$, of bandwidth $[F(3,69)=7.8, p=.0003]$, and of their interaction $[F(9,207)=5.4, p<.00001]$. It is important to notice that in the conditions in which the effects of BW are clearest (where the separation of the band edges is 30 semitones), as BWs get smaller, the CF separations between $\mathrm{H}$ and $\mathrm{L}$ automatically get larger. We observe that the corresponding rated segregation also gets larger. This means that it is correlated with $C F$ separation rather than with BW. So the results could be attributed to CF separation alone.

\section{Series B}

Analysis of the inner band edge separation by BW (second part of Table 1 and middle panel of Figure 2) showed strong effects of inner band edge separation $[F(3,69)=$ $231.8, p<.00001]$ and bandwidth $[F=114.3, p<.00001]$, as well as their interaction $[F(9,207)=7.44, p<.00001]$. The interaction seems due to the fact that the strongest effects of BW are found at the two middle levels of inner band edge separation, where CFs run from 4 to 13 semitones. Notice that in this series of conditions, BW and CF separation are positively correlated and the rated segregation is correlated with both. So, again, the results could be attributed to CF separation alone.

\section{Series C}

Analysis of CF separation by BW (third part of Table 1 and lower panel of Figure 2) showed that the CF separation effect had an extremely low probability of occurring by chance $[F(3,69)=233.9, p<.00001]$, but BW, which for the first time was not correlated with $\mathrm{CF}$, showed no significant effect $[F(3,69)=0.83, p=.48]$; nor was there a significant $\mathrm{CF} \times \mathrm{BW}$ interaction $[F(9,207)=0.50$, $p=.87]$. Thus, when $\mathrm{BW}$ was uncorrelated with $\mathrm{CF}$, the ANOVA showed no effect of $\mathrm{BW}$ on segregation, but $\mathrm{CF}$ separation still showed a powerful effect. Inspection of the Series $C$ results shown in Table 1 and Figure 2 shows that the effect of CF was not only significant but also very large. If either outer band edges or inner band edges influenced segregation, then as BW increased in Series C, the correlated increase in the separation of the inner and the outer band edges should have caused a change in the responses of the listeners that would be have been interpreted as a BW effect. Unless the effects of inner and outer band edges exactly compensated for one another, a BW effect should have been observed. (Saying that they compensate for one another is equivalent to saying that only $\mathrm{CF}$ controls segregation.)

To get an indication of the effects of $\mathrm{CF}$ and $\mathrm{BW}$ across the three series of conditions, we ran a multiple regression analysis using CF and BW as predictor variables and the average rated difficulty of integration of each condition (called "rating") as the dependent variable. The simple correlations are as follows: $\mathrm{CF}$ with rating, $r=.90 ; \mathrm{BW}$ with rating, $r=.14$; and $\mathrm{CF}$ with $\mathrm{BW}, r=.00$. (This last correlation follows mathematically from the design of the experiment.) A stepwise multiple regression showed that when $\mathrm{CF}$ was in the predictor equation alone, multiple $R$ was .90 and the proportion of variance accounted for was $80.5 \%(t=14.39, p<.00001)$. Adding the BW variable increased multiple $R$ by only .01 to .91 , adding only $2 \%$ to the accounted-for variance $(t=2.28, p=$ $.03)$. Hence the most powerful predictive effect came 


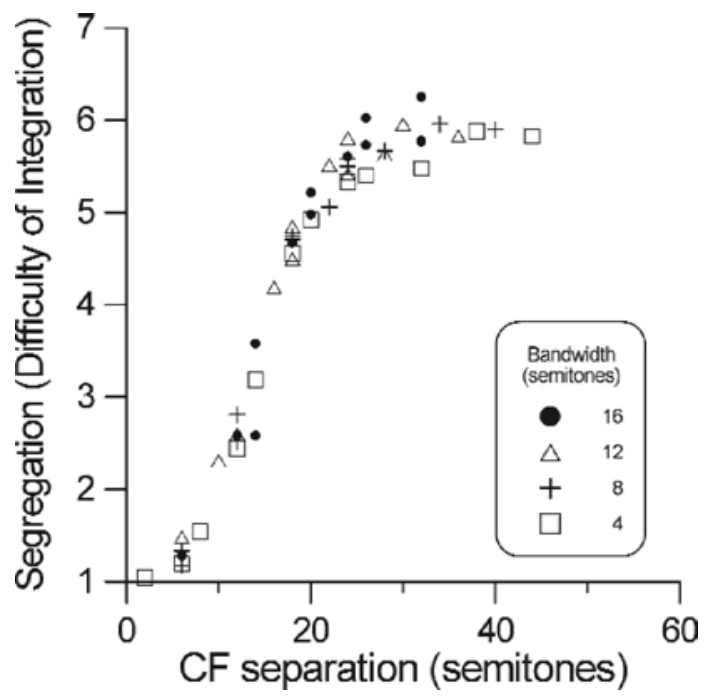

Figure 3. Segregation, as measured by rated difficulty of integration $(1=e a s y, 7=$ hard $)$, is plotted as a function of center frequency (CF) separation in semitones. Each point represents the mean for all listeners for 1 of the 48 experimental conditions. High scores represent compelling segregation. The geometric symbols show the bandwidths; the two bursts that were alternated with each other always had the same bandwidth (in semitones).

from CF with only a very small contribution from BW. The direction of the BW effect was such that a larger BW made it a little harder to hold on to the sequence as a single integrated stream.

The overall effects of $\mathrm{CF}$ on ratings can be seen in Figure 3, which plots all the ratings given for Series A, B, and $\mathrm{C}$ as a function of $\mathrm{CF}$ separation. Each point represents the mean for all listeners for one of the 48 experimental conditions. The parameter is the BW in semitones. (The fact that there is more than one condition with a given $\mathrm{CF}$ separation and $\mathrm{BW}$, i.e., two identical symbols at the same value of $X$, is not a mistake, but resulted from the nonorthogonal design of the experiment.) It can be seen that the variations in BW had an inconsistent and relatively small effect. The curve is not linear on a semitone scale: There is a fairly rapid change in the ratings of difficulty of integration as CF separation increases between 8 and 24 semitones. Segregation ratings then increase more and more slowly, with an asymptote at about 6 on the 7-point scale. Even with this curvature, the correlation (which looks only at the linear component of the covariation between $\mathrm{CF}$ and rating of difficulty of integration) was .90 .

The results for BW, then, were that in Series C, where it was manipulated independently of $\mathrm{CF}, \mathrm{BW}$ showed no significant effect, but when the three series of conditions were combined in a regression analysis, it showed a weak positive correlation with segregation.

We were unable to show that looking at frequency separation in terms of inner or outer band edges was superior to viewing it in terms of $\mathrm{CF}$ and $\mathrm{BW}$. The latter classification of the data (Series 3 conditions) gave the clearest account, leaving no interactions to explain. This suggests that the separation in frequency between two bands of noise that are flat in loudness (Fletcher-Munson corrected amplitude) between two sharp band edges can be well described in terms of CF computed on a log scale.

\section{DISCUSSION}

It is not entirely clear whether the BW effect is real. In the results of Bregman et al. (1999), BW showed a significant effect on rated stream segregation $(p<.0001)$. This variable affected the response measure to the extent of about 1 point on a 7-point scale, whereas in the present experiment $\mathrm{BW}$ had a minimal effect $(p=.48$ in the analysis of Series 3 conditions, and $p=.03$ in the multiple regression). Recall that the effects of the $\mathrm{BW}$ variable in the experiment of Bregman et al. can be interpreted as resulting from differences in intensity-hence in loudness - that were introduced automatically when the $\mathrm{H}$ bands were made wider that the $\mathrm{L}$ bands in order to equate higher and lower bands on their "pitch strengths." These intensity differences could not be eliminated, due to other constraints in their experiment. In the present experiment, the widths of the $\mathrm{H}$ and $\mathrm{L}$ bands were first equalized on a log-frequency scale to equate for pitch strength, then individually attenuated to obtain equal RMS amplitude levels (prior to Fletcher-Munson loudness compensation). This may account for some or all of the difference between the BW results in the experiment by Bregman et al. and the present one.

Another difference between the experiment of Bregman et al. (1999) and the present one is that in the earlier experiment, the $\mathrm{CF}$ of a band was defined as the arithmetic mean of the band of frequencies, whereas in the present experiment, $\mathrm{CF}$ was defined as its geometric mean frequency (logarithmic mean frequency). In the present experiment, perceived segregation depended almost entirely on the difference in CF between $\mathrm{L}$ and $\mathrm{H}$ bands when the difference in $\mathrm{CF}$ was measured in semitones. However, in Bregman et al., where CF was defined as the arithmetic mean of the band, there was an effect of $\mathrm{BW}$ in addition to the effect of CF. Because the value of the $\mathrm{CF}$ for any noise band changes when we switch from an arithmetic to a geometric mean frequency, this might lead one to hypothesize that the effect of BW would not be as strong in the earlier experiment if $\mathrm{CF}$ were to be measured using geometric means. However, this difference in definition cannot account for the stronger BW effect in Bregman et al. for the following reason. In Bregman et al., the BWs of the $\mathrm{L}$ and $\mathrm{H}$ noises were approximately equal on a logarithmic scale. When $\mathrm{L}$ and $\mathrm{H}$ BWs are exactly equal on a logarithmic scale, the ratio of the arithmetic mean of the higher band to the arithmetic mean of the lower band is identical to the ratio of the geometric means of the same two bands. In other words, two noises whose BWs are equal on a logarithmic scale will be separated by the same number of semitones independently of whether one uses arithmetic means or geometric means 
to measure CF. Therefore, the small BW effect seen in Bregman et al. cannot be due to this difference in CF definition, and is more likely to be a consequence of the inequalities in the intensities of the $\mathrm{H}$ and $\mathrm{L}$ bands in that experiment, as noted.

Nevertheless, it would be interesting to determine whether a linear measure of frequency difference would provide a better or worse predictor of segregation than a logarithmic measure of frequency separation (number of semitones). Accordingly, we repeated the regression analysis using the arithmetic difference in $\mathrm{CF}$ (in $\mathrm{Hz}$ ) and the arithmetic BW (in $\mathrm{Hz}$ ) as predictors. The variance accounted for by the regression was less than in the original $\log$-frequency (semitone scale) analysis. Multiple $R$ for the linear analysis was only .837 (accounting for $70 \%$ of the variance among conditions), as compared with the value of .908 in the log-frequency analysis (accounting for $82 \%$ of the variance). Correspondingly, the correlation between the linear $\mathrm{CF}$ and the response measure was only .837 , versus .897 in the earlier analysis. This suggests that segregation is actually controlled by some measure of frequency separation between $\mathrm{H}$ and $\mathrm{L}$ stimuli that is more closely approximated by a semitone (a log-frequency) scale than by a linear frequency scale, when noise BWs are equivalent on a log scale.

Figure 3 shows that beyond an approximately twooctave separation between the CF of high and low NBNs, there is no further increase in the difficulty of holding on to a single stream. In a review of this paper, Pierre Divenyi (personal communication, December 1998) pointed out that similar observations have been made for the discrimination of unfilled intervals between two tones of different frequencies (Divenyi \& Danner, 1977) and for the detection of gaps between narrow-band noise-burst markers (Formby, Barker, Abbey, \& Raney, 1993). The similarity suggests to Divenyi that all these observations may be looking at the same process: frequency integration in temporal processing.

A final point of interest is a comparison of the effects of frequency separation upon stream segregation for narrow-band noises (sharply peaked, falling off by $48-\mathrm{dB} /$ octave on both high and low sides) and pure tones. Some of the conditions of Dannenbring and Bregman (1976) permit such a comparison. Table 2 shows the relevant conditions, reproduced from that paper. The scores were ratings on a 14-point scale running from 1 (very confident one stream) to 14 (very confident two streams). There were two values of frequency separation: $200 \mathrm{~Hz}$ (3.2 semitones) and $1000 \mathrm{~Hz}$ (19 semitones); for the tones, this was the separation between their actual frequencies, and for the noise bands, the separation between their spectral peaks. There were two different values of interstimulus interval (ISI), the duration of the silence between the end of one sound and the start of the next: namely 15 and $30 \mathrm{msec}$. There were also two values of stimulus onset asynchrony (SOA) - the time from the onset of one sound to the onset of the next: namely 135 and $185 \mathrm{msec}$. For each of the resulting four combinations of ISI with SOA, the increase in frequency separation between the high and low sounds caused the segregation to become stronger. This effect was at least as strong, overall, for noise bursts as for pure tones (see the final column of Table 2). However, the timing factors seemed to interact differently with frequency separation for the noises and the tones. For an SOA of $135 \mathrm{msec}$, the effects of frequency separation seemed to be much stronger for noise bursts than for pure tones. However, for an SOA of $185 \mathrm{msec}$, the effects of frequency separation seemed to be stronger for the tones than for the noise bursts. Any explanation for this discrepancy awaits further research.

In conclusion, we were able to account for the segregation of $\mathrm{H}$ and $\mathrm{L}$ noise bands quite well by the separation between their CFs, where CF is defined as the halfway point, in log frequency, between the upper and lower band edges. However, it is important to recognize the limitations of this finding. Our signals were idealizations of natural noise bursts. It is not obvious that one could predict the integration or segregation of sequences of noises encountered in everyday life by knowing only their CFs on a $\log$ scale. For example, the spectra of our noise bands were flat between band edges and therefore were symmetrical in log frequency around the CF (prior to loudness equalization). There was also no energy in the signal outside these bands. We do not yet know how to get a measure for nonflat spectra that would be as good a predictor as CF was in the present experiment. Nor do we know whether such a CF measure is even relevant in cases in which each spectrum has more than one peak in it.

Table 2

Results From Dannenbring and Bregman (1976)

\begin{tabular}{|c|c|c|c|c|c|}
\hline & \multicolumn{2}{|c|}{ ISI $15 \mathrm{msec}$} & \multicolumn{2}{|c|}{ ISI $30 \mathrm{msec}$} & \multirow[b]{2}{*}{$M$} \\
\hline & SOA $135 \mathrm{msec}$ & SOA $185 \mathrm{msec}$ & SOA $135 \mathrm{msec}$ & SOA $185 \mathrm{msec}$ & \\
\hline \multicolumn{6}{|l|}{ Noise conditions } \\
\hline 1,000 with $1200 \mathrm{~Hz}$ & 3.95 & 5.85 & 2.35 & 1.85 & 3.50 \\
\hline 1,000 with $3000 \mathrm{~Hz}$ & 12.45 & 10.25 & 11.35 & 5.45 & 9.88 \\
\hline \multicolumn{6}{|l|}{ Tone conditions } \\
\hline 1,000 with $1200 \mathrm{~Hz}$ & 7.60 & 3.20 & 6.30 & 2.65 & 4.93 \\
\hline 1,000 with $3000 \mathrm{~Hz}$ & 10.55 & 10.40 & 10.40 & 9.10 & 10.11 \\
\hline
\end{tabular}


Second, the characteristics of our noise bands were stationary over time (except for the random variation inherent in noise). If they were to change over time, we would not know the appropriate way to combine the very-shorttime spectra within a changing noise burst to derive a summary frequency for the burst that would allow us to predict its grouping with a subsequent burst. The particular type of change over time is probably also significant. We know that in the case of pure tone glides, the slope is important: A glide, B, will more readily group with a preceding glide, $\mathrm{A}$, of the same $\mathrm{CF}$ when $\mathrm{A}$ and $\mathrm{B}$ are identical than when $\mathrm{B}$ is a temporal reversal of $\mathrm{A}$ and therefore has the "opposite" slope (Steiger \& Bregman, 1981). Furthermore, surely the measure of frequency similarity should depend on the durations of the bursts in question. For example, how heavily should any estimate of a frequency-based grouping tendency weight the parts of the two bursts that are closer in time to one another versus the parts that are temporally more remote? How far away in time do the parts of two successive noise bursts have to be before they do not affect the grouping at all? These questions would have to be answered before the grouping of noises in speech, music, or artificial auditory displays could be predicted with any accuracy.

\section{REFERENCES}

Bregman, A. S. (1990). Auditory scene analysis: The perceptual organization of sound. Cambridge, MA: MIT Press.

Bregman, A. S., Colantonio, C., \& Ahad, P. A. (1999). Is a common grouping mechanism involved in the phenomena of illusory continuity and stream segregation? Perception \& Psychophysics, 61, 195-205.

Dannenbring, G. L., \& Bregman, A. S. (1976). Stream segregation and the illusion of overlap. Journal of Experimental Psychology: Human Perception \& Performance, 2, 544-555.

Divenyi, P. L., \& DANner, W. F. (1977). Discrimination of time intervals marked by brief acoustic pulses of various intensities and spectra. Perception \& Psychophysics, 21, 125-142.

Formby, C., Barker, C., Abbey, H., \& Raney, J. J. (1993). Detection of silent temporal gaps between narrow-band noise makers having second-formantlike properties of voiceless stop/vowel combinations. Journal of the Acoustical Society of America, 93, 1023-1027.

HENKE, W. L. (1990). MITSYN: A synergistic family of high-level languages for time signal processing (Version 8.1). Belmont, MA: Author.

Steiger, H., \& Bregman, A. S. (1981). Capturing frequency components of glided tones: Frequency separation, orientation, and alignment. Perception \& Psychophysics, 30, 425-435.

VAN NoORden, L. P. A. S. (1975). Temporal coherence in the perception of tone sequences. Unpublished doctoral dissertation, Eindhoven University of Technology.

(Manuscript received September 2, 1998; revision accepted for publication October 15, 2000.) 